TP Periodica Polytechnica

Electrical Engineering and Computer Science

58(2), pp. 37-42 (2014)

DOI:10.3311/PPee.7024

Creative Commons Attribution (1)

RESEARCH ARTICLE

\section{The Effect of Latency Increasing on the Realisation Cost in High Level Synthesis of Pipeline Systems}

\author{
György Pilászy / György Rácz / Péter Arató
}

RECEIVED 27 August 2013; ACCEPTED AFTER REVISION 25 February 2014

\section{Abstract}

This paper examines the effects of increasing the latency in pipeline systems. The high level synthesis methods focus on the pipeline throughput only, and the latency is an output parameter. The proposed method is capable for reducing the cost of the implementation by increasing the latency at the same throughput. The essence of the proposed method is that an increase in the latency may increase the mobility ranges of the processing units. Thus, the increased degrees of freedom may cause better implementation by affecting the scheduling and allocation steps. An impact assessment algorithm for calculating proper latency increment range is also presented.

\section{Keywords}

Pipelining $\cdot$ latency time $\cdot H L S \cdot C A D \cdot$ microcontroller . multiprocessing $\cdot$ embedded systems

\section{György Pilászy}

Department of Control Engineering and Information Technology, Faculty of Electrical Engineering and Informatics, BME,

Magyar tudósok krt 2., H-1117 Budapest, Hungary

e-mail: gpilaszy@iit.bme.hu

\section{György Rácz}

Department of Control Engineering and Information Technology, Faculty of Electrical Engineering and Informatics, BME,

Magyar tudósok krt 2., H-1117 Budapest, Hungary e-mail: gyuriracz@iit.bme.hu

\section{Péter Arató}

Department of Control Engineering and Information Technology, Faculty of Electrical Engineering and Informatics, BME,

Magyar tudósok krt 2., H-1117 Budapest, Hungary

e-mail: arato@,iit.bme.hu

\section{Introduction}

During the high-level synthesis of the pipeline systems, the most important parameter is the throughput $[1,3,6]$. The effect of increasing the latency period is not analysed. However, a longer latency does not slow down the system in pipeline sense, since the effect is negligible for the throughput.

One of the aims of this paper is to determine the effect of increasing the latency to the cost function in the pipeline systems. For demonstration, PIPE HLS tool (developed by BME-IIT) has been used in the analysis, but the results are not affected by using commercial tools (e.g. Catapult C). The cost reduction is expected, since the extent of overlapping between the elementary operations may be reduced by increasing the latency. Thus, the scheduling and allocation steps of HLS may result in a less expensive structure by taking advantage of better reusing situation. An illustration is shown in Figure 1. The processing of the first data is marked in (red) dark, and of the second data in (blue) bright. The unidirectional arrows represent the data dependence. For simplicity, let the $e_{1} \ldots e_{5}$ elementary operations be identical. The processing time of each elementary operation is assumed 8 clock cycles. The elementary operations $e_{2}$ and $e_{3}$ start and finish at the same time. Thus they can not be implemented in the same processing unit. The overlapping will be eliminated, for example, if $e_{2}$ starts at a later time $\left(t_{3}\right)$. This case is shown in Figure 2. It can be observed that the latency has increased, because of the execution time of $e_{2}$ and the data dependency.

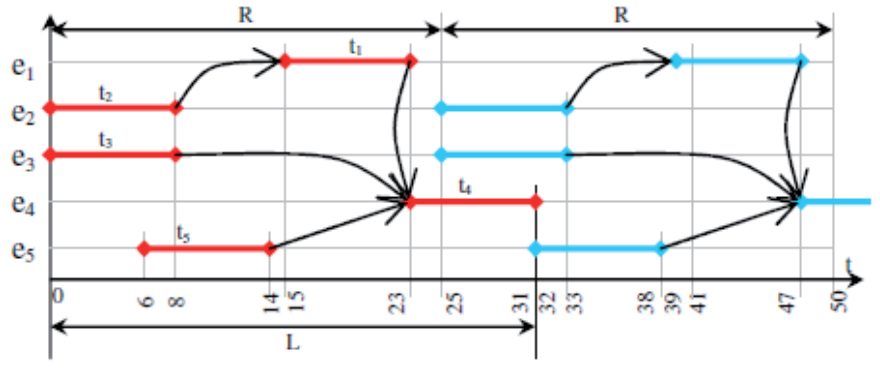

Fig. 1. Scheduling of a pipeline system $(L=31, R=25)$ 
Since one processing unit can perform at most three elementary operations within the specified restart time $(3 \cdot 8<R=25)$, therefore at least two processing units should be used. This situation is shown in Figure 2. The elementary operations $e_{1}, e_{2}, e_{3}$, and $e_{4}, e_{5}$ can be allocated into two processing units, respectively. In consequence, the latency is increased by $1(L=32)$.

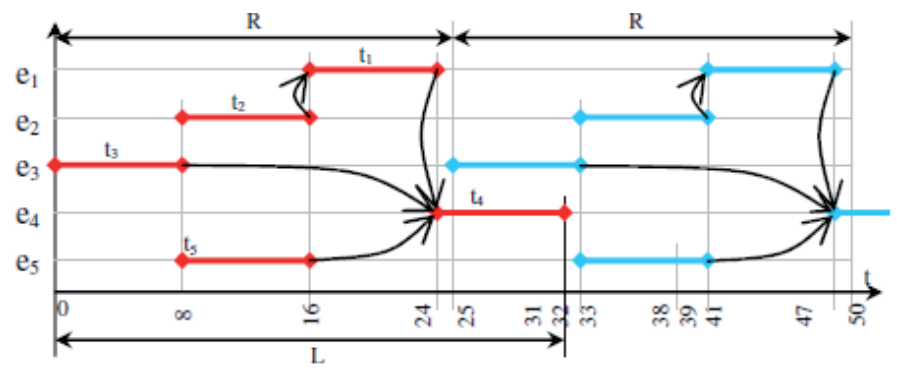

Fig. 2. Scheduling with increased latency $(L=32, R=25)$

A further reduction of the required number of processing units may be achieved by applying longer restart time. An extreme situation is illustrated in Figure 3. This solution requires only one processing unit, because there are no overlapped elementary operations, but there is no pipelining any more $(R=L)$.

The organization of the remaining sections is as follows. In section 2, a simple cost function will be assumed for illustrating the effect of the longer latency, and then simulation results are presented as case studies. In section 3, an algorithm is described for calculating the increased latency. The paper ends with the conclusion in section 4 .

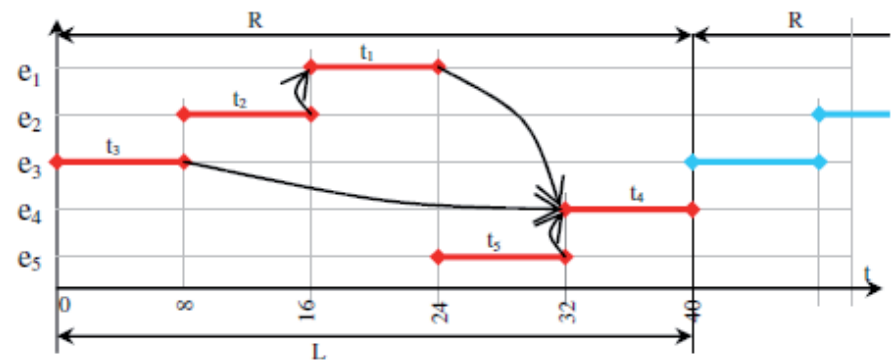

Fig. 3. Scheduling with increased restart time and latency $(L=40, R=40)$

\section{Case studies}

In this section, we present examples to illustrate the cost reduction effect of the increased latency. Firstly, we define our assumed simple cost function.

\subsection{The simple cost function}

In our example, each node of the input graph contains one elementary operation. Let $t_{i}$ denote the execution or processing time of the elementary operation $e_{i}$. After the scheduling and allocation steps, more elementary operations can be allocated into one processing unit $\left(P_{i}\right)$. The aim is to minimize the number of these processing units. Let $C_{i}$ denote the cost of processing unit $P_{i}$. If the number of processing unit $P_{i}$ is $n_{i}$

$$
C_{i}=n_{i} \cdot t_{i}
$$

The simple total cost $(C)$ is the sum of partial costs $C_{i}$ :

$$
C=\sum_{\forall i} C_{i}=\sum_{\forall i} n_{i} \cdot t_{i}
$$

By calculating this cost for the examples of Figures 1-3, the results are shown in Table 1.

Tab. 1. The costs calculated for Figures $1 . .3$

\begin{tabular}{cccc}
\hline $\boldsymbol{R}$ & $\boldsymbol{L}$ & $\boldsymbol{n}_{\boldsymbol{i}}$ & $\boldsymbol{C}$ \\
\hline 25 & 31 & 3 & 24 \\
25 & 32 & 2 & 16 \\
40 & 40 & 1 & 8 \\
\hline
\end{tabular}

There are two ways for cost reduction by increasing the latency. The simplest way is to increase the latency step-by-step by one, to execute the whole HLS software each time and to check the new total cost value. The disadvantage of this method is the great number of re-run steps that takes too much time. The other way is to determine only those latency increasing steps in advance, which might decrease the total cost. Some rules can be formulated for finding these favourable latency increasing steps as follows.

If the same types of elementary operations start simultaneously, then the latency should be increased at least by the value of the execution time in order to allocate them into the same processing unit. For reusing a processing unit $\left(P_{i}\right) \mathrm{k}$ times within a restart period $(R)$, it is necessary that the overall execution time of elementary operations allocated into $P_{i}$ must not be greater than the restart time $(R)$ :

$$
\sum_{k} t_{k} \leq R
$$

If the execution times of all elementary operations allocated in $P_{i}$ are identical, then

$k \cdot t_{k} \leq R$ must hold.

Further on, the above procedure is illustrated by a simple benchmark from [1]. 


\subsection{FFT algorithm}

In the following example [1], there is an eight-point FFT (Fast Fourier Transformation) algorithm, the elementary operation graph of which is shown in Figure 4. In Table 2, the assumed execution times are summarized.

Tab. 2. Elementary operations of the FFT

\begin{tabular}{cccc}
\hline Execution units & Operation & $\mathrm{t}_{\mathrm{i}}$ & pieces \\
\hline P1 & MUL & 12 & 12 \\
P2 & SUB & 3 & 12 \\
P3 & ADD & 3 & 12 \\
\hline
\end{tabular}

The initial value of the latency is $L=48$. This is calculated by PIPE from the original graph for $R=10$ restart time. Since the shortest elementary operation time $\left(t_{i}\right)$ is 3 , the restart period should be at least $R>6$ in order to achieve a beneficial effect by increasing $L$.

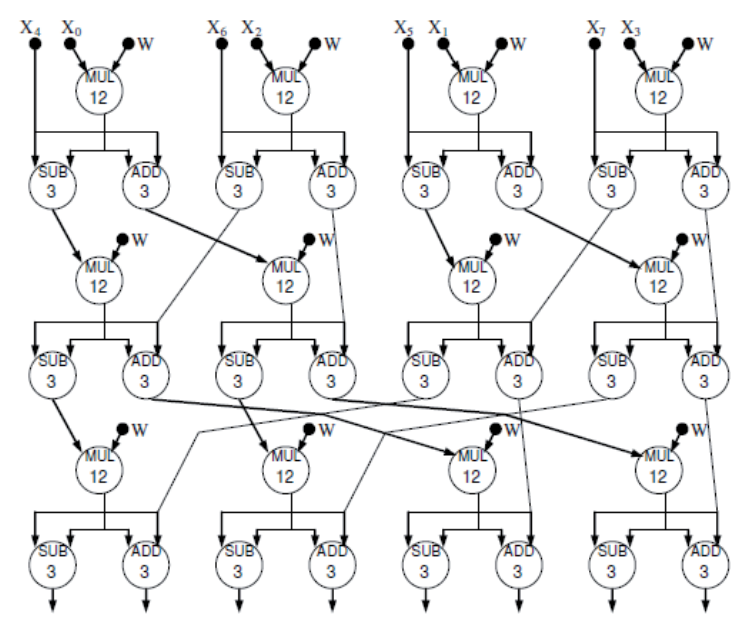

Fig. 4. Elementary operation graph of the FFT algorithm

The time range of scanning the restart period: is chosen for $R=\{12, \ldots, 49\}$

The range of the latency value: $L=\{48,51,54,60,96\}$

Table 3 shows the cost functions based on Table 2. The cost functions are shown in Figure 5. The procedure has been carried out also in case of multifunction execution units assumed to be able to execute both SUM and ADD elementary operations. Table 4 shows the number of required multifunction execution units. The cost functions are shown in Figure 6.

It can be observed, that the cost values are not always smaller at the longest latency. The reason of this is the approximate character of the scheduling algorithm actually applied in the HLS tool. Namely, the start times of the elementary operations are fixed somehow depending on each other, and so sometimes stricter constraints may arise for the allocation algorithm. In the above examples, the force-directed scheduler of the HLS tool PIPE was applied.

\subsection{Observations:}

1. Increasing the latency value increases the mobility of the elementary operations. The larger mobility increases the running time of the scheduling and allocating algorithm because of the higher degrees of freedom. However, this higher degree of freedom may give better results.

2. If $R \leq 2 \cdot \min \left\{t_{j}\right\}$, then no significant decrease of the cost can be expected, because of the replication and so with frequent restarting, none of the execution units can be reused.

3. If an $e_{i}$ elementary operation has the execution time $t_{i} \geq R$, then no significant improvement can be expected in the number of the units executing $e_{i}$, because of the replication performed by the HLS tool [1].

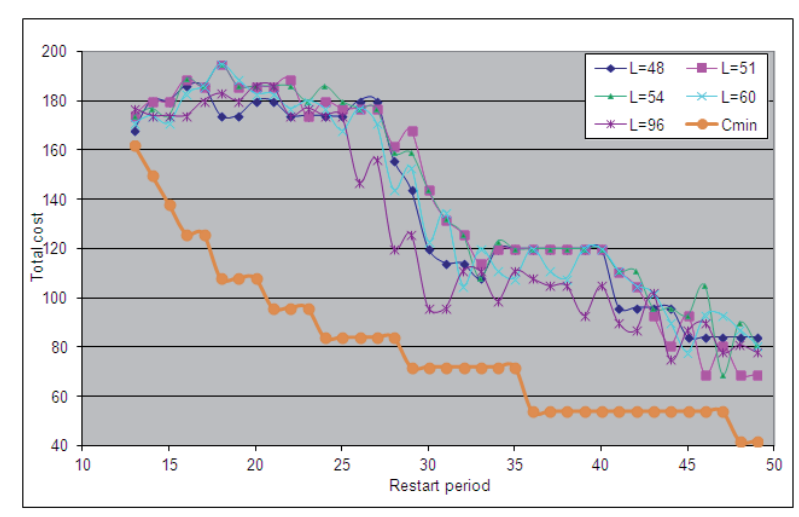

Fig. 5. Cost function of the FFT algorithm

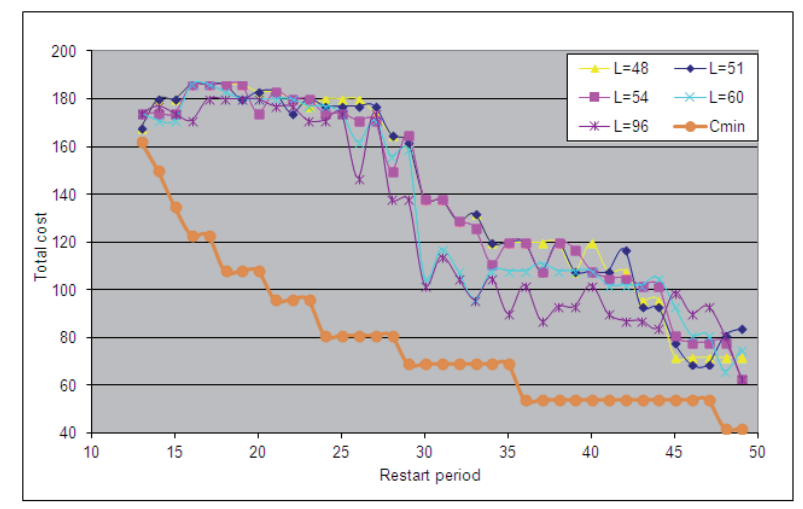

Fig. 6. Cost function of the FFT algorithm in case of multifunction execution units

\section{The proposed algorithm}

Let the following notations be introduced:

$L_{\text {min }}$ : the latency value determined by the HLS tool for the desired restart time $R$ 
$L_{\text {max }}$ : an arbitrary value (greater than $L_{\text {min }}$ ) defined by the user, typical value is $2 \cdot L_{\min }$

$e_{j}$ : an elementary operation (a node) in EOG, the range of $\mathrm{j}$ consists of the integers from 1 until the number of nodes in $\mathrm{EOG}$

$E\left\{e_{1}, \ldots e_{j,} \ldots e_{m}\right\}$ : The set of the elementary operations in EOG. $\Phi_{F}\left\{f_{l}, f_{2}, \ldots, f_{k}, \ldots f_{n}\right\}:$ A complete cover of the elementary operations, where block $f_{k}$ contains all elementary operations with type $k$. (It is cover, because one $e_{j}$ can be executed by more than one execution unit.)

$t_{k}$ : the execution time of the elementary operations belonging to block $f_{k}$. (this value is identical for all operations belonging to the same block).

$n_{k}$ : the number of the elementary operations in block $f_{k}$

$P_{i}$ : the execution unit type $i$ defining an incomplete cover on $\Phi_{F}$ denoted by $\Phi_{i}$, Te blocks of $\Phi_{i}$ contain those elementary operations from the blocks of $\Phi_{F}$, which are executable by $P_{i}$

$M_{q}$ : the $q$-th copy of an execution unit after the allocation (its type is determined by a $P_{i}$

$M\left\{M_{1}, \ldots, M_{q}, \ldots M_{z}\right\}:$ the set of the necessary copies of the execution units determined by the allocation.

$\Pi_{M}\left\{m_{1}, \ldots, m_{i}, \ldots m_{p}\right\}$ : the complete partition of the execution unit copies. (Block $\mathrm{m}_{\mathrm{i}}$ contains all those copies $M_{q}$, the type of which is determined by $P_{i}$.

$N_{i}$ : the number of copies in bolck $m_{i}$, i.e. the number of neccessary copies of $P_{i}$.

$T_{i}$ : the longest execution time of elementary operations which are executable by $P_{i}$.

$C_{i}$ : The cost of applying execution unit $P_{i}$ determined by the allocation: $C_{i}=N_{i} \cdot T_{i}$

$C:$ the total cost: $C=\sum_{i} C_{i}$

$C_{d}$ : a minimal cost value, defined by the user for stop.

$N_{\text {imin }}$ : the minimal number of $P_{i}$ copies for a given restart time

$R$. This value of $R$ could not be achieved by applying less copies of $P_{i}$

$C_{\text {min }}:$ the minimal cost $C_{\text {min }}=\sum_{i} \mathrm{~N}_{\text {imin }} \cdot T_{i}$

\subsection{The main steps of the algorithm:}

1. Determine the minimal value of the latency time $\left(L_{\min }\right)$ by the HLS tool. [1]

2. Determine to each $P_{i}$ belong the minimal number of the execution units (rounded up).

$$
N_{i M I N}=\sum_{k}\left\lceil\frac{n_{k} \cdot t_{k}}{R}\right\rceil
$$

3. Determine the minimal cost $\left(C_{\text {min }}\right)$. In case of fixed restart time $(R)$, the lowest possible cost can be estimated by the following equation:

$$
C_{\min }=\sum_{\forall i} N_{i M I N} \cdot T_{i}
$$

If the value of $\mathrm{R}$ can be increased, then the theoretical minimum cost is obtained when each elementary operation occur as few times as possible: $n_{1}=n_{2}=\ldots n_{i}=1$. Then the minimum cost value:

$$
C_{\min }=\sum_{\forall i} T_{i}
$$

This result represents already a non pipeline situation, because the elementary operations do not overlap each other: $R \geq L$.

4. Create sets $M\left\{M_{1}, M_{q}, \ldots, M_{z}\right\}$ of execution units after the allocation. To each $M_{q}$ belongs the execution time $t_{i}$.representing the longest execution time of the elementary operations executed by the execution units in $M_{i}$.

5. Create $\Pi_{M}\left\{m_{1}, \ldots, m_{i}, \ldots, m_{p^{\prime}}\right\}$ full partition of $M$ according to the $P_{i}$ then select those, where $\left|m_{i}\right|>N_{i M I N}$ and $t_{i} \leq R / 2$

6. Select the smallest $t_{i}$ for incrementing $L$ according to the $\max \left(\left|m_{i}\right|\right)$

7. Increase the latency by the selected Ti and rerun the HLS tool. (go to step 4.)

8. The new latency $L+t_{i}$ yields a new cost value $C$

9. There might be set a limit $L_{\max }$ of incrementing the latency. A possible default value could be: $L_{\max }=2 \cdot L_{\min }$.

The stopping condition can be formulated in many ways. One possibility is that we stop the iteration when we reached the predefined maximum latency value $\left(L_{\max }\right)$. This condition can be tested easily.

Another possibility is that the iteration is continued until the theoretically achievable minimum $\operatorname{cost}\left(C_{\min }\right)$ or a desired cost $\left(C_{d}\right)$ is reached.

The combination of both methods can give good results. In each iteration step, it should be examined which of the above conditions is achieved $\left(L_{\max }\right.$ or $\left.C_{\min }\right)$. If any of these conditions are fulfilled, the iteration would be stopped.

\section{Conclusion}

The proposed method can reduce the cost of hardware implementation of a task by increasing the latency time in pipeline systems while maintaining the same throughput. The method helps in designating efficient ranges for incrementing the latency, and enables the impact assessment of a range regarding the total cost. 


\section{Acknowledgement}

The support of the Hungarian Scientific Fund (OTKA K72611) and New Hungary Development Plan (Project ID: TÁMOP4.2.1/B-09/1/KMR-2010-0002) IKT-P5-T3 are gratefully acknowledged. This work is also belonging to the scientific program of the "Development of quality-oriented and harmonized R+D+I strategy and functional model at BME" project.

\section{References}

1 Arató P., Visegrády T., Jankovits I., High Level Synthesis of Pipelined Datapaths. New York: John Wiley \& Sons, (2001).

2 Pilászy Gy., Móczár G., Remote control of modular microcontroller systems, Microcad 2001. In: International Scientific Conference Section F: Measurement and Automation. Miskolc, 1th March 20012th March 2001, pp. 45-50, (2001).

3 Arató P., Drexler D., Kocza G., Suba G., Synthesis of a Taskdependent Pipelined Multiprocessing Structure. ACM Transactions on Design Automation of Electronic Systems. (submitted to)

4 Coussy P., Gajski D. D., Meredith M., Takach A., An Introduction to High-Level Synthesis. IEEE Design \& Test of Computers, 26 (4), pp. 8-17, (2009). DOI: $\underline{10.1109 / M D T .2009 .69}$

5 Pilászy Gy., Rácz Gy., Arató P., Communication Time Estimation in High Level Synthesis. Periodica Polytechnica Electrical Engineering and Computer Science, 57 (4), pp. 99-103, (2013). DOI: $10.3311 /$ PPee. 7413

6 Fingeroff M. High-Level Synthesis Blue Book. Xlibris Corporation, (2010).

7 Xu J., Wolf W., Henkel J., Chakradhar S., A design methodology for application-specific networks-on-chip. ACM Transactions on Embedded Computing Systems, 5 (2), pp. 263-280, (2006). DOI: $10.1145 / 1151074.1151076$

8 Mann Z. Á., Orbán A., Arató, P., Finding optimal hardware/software partitions. Formal Methods in System Design, 31 (3), pp. 241-263, (2007). DOI: $10.1007 / \mathrm{s} 10703-007-0039-0$

9 Arató P., Mann Z. Á., Orbán A., Algorithmic aspects of hardwarel software partitioning. ACM Transactions on Design Automation of Electronic Systems, 10 (1), pp. 136-156, (2005). DOI: $10.1145 / 1044111.1044119$

10 Suba G., Hierarchical pipelining of nested loops in high-level synthesis. submitted to Periodica Polytechnica Electrical Engineering and Computer Science, 58, (2014). DOI: $10.3311 /$ PPee.7610 
Tab. 3. Total cost of execution units of the FFT algorithm

\begin{tabular}{|c|c|c|c|c|c|c|}
\hline$\propto$ & $\stackrel{\infty}{\mathbb{1}}$ & i⿱ & II & ت્ & 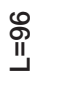 & $U^{\frac{\bar{E}}{E}}$ \\
\hline 13 & 168 & 174 & 174 & 171 & 177 & 162 \\
\hline 14 & 180 & 180 & 177 & 174 & 174 & 150 \\
\hline 15 & 180 & 180 & 174 & 171 & 174 & 138 \\
\hline 16 & 186 & 189 & 189 & 183 & 174 & 126 \\
\hline 17 & 186 & 186 & 186 & 186 & 180 & 126 \\
\hline 18 & 174 & 195 & 195 & 195 & 183 & 108 \\
\hline 19 & 174 & 186 & 186 & 189 & 180 & 108 \\
\hline 20 & 180 & 186 & 186 & 183 & 186 & 108 \\
\hline 21 & 180 & 186 & 186 & 183 & 186 & 96 \\
\hline 22 & 174 & 189 & 186 & 177 & 174 & 96 \\
\hline 23 & 174 & 174 & 180 & 180 & 177 & 96 \\
\hline 24 & 174 & 180 & 186 & 177 & 174 & 84 \\
\hline 25 & 174 & 177 & 180 & 168 & 174 & 84 \\
\hline 26 & 180 & 177 & 177 & 177 & 147 & 84 \\
\hline 27 & 180 & 177 & 177 & 171 & 156 & 84 \\
\hline 28 & 156 & 162 & 159 & 144 & 120 & 84 \\
\hline 29 & 144 & 168 & 159 & 153 & 126 & 72 \\
\hline 30 & 120 & 144 & 144 & 123 & 96 & 72 \\
\hline 31 & 114 & 132 & 132 & 135 & 96 & 72 \\
\hline 32 & 114 & 126 & 126 & 105 & 111 & 72 \\
\hline 33 & 108 & 114 & 108 & 120 & 111 & 72 \\
\hline 34 & 120 & 120 & 123 & 111 & 99 & 72 \\
\hline 35 & 120 & 120 & 120 & 108 & 111 & 72 \\
\hline 36 & 120 & 120 & 120 & 120 & 108 & 54 \\
\hline 37 & 120 & 120 & 120 & 111 & 105 & 54 \\
\hline 38 & 120 & 120 & 120 & 108 & 105 & 54 \\
\hline 39 & 120 & 120 & 120 & 120 & 93 & 54 \\
\hline 40 & 120 & 120 & 120 & 120 & 105 & 54 \\
\hline 41 & 96 & 111 & 111 & 111 & 90 & 54 \\
\hline 42 & 96 & 105 & 111 & 105 & 87 & 54 \\
\hline 43 & 96 & 93 & 96 & 102 & 102 & 54 \\
\hline 44 & 96 & 81 & 96 & 90 & 75 & 54 \\
\hline 45 & 84 & 93 & 93 & 78 & 87 & 54 \\
\hline 46 & 84 & 69 & 105 & 93 & 90 & 54 \\
\hline 47 & 84 & 81 & 69 & 93 & 78 & 54 \\
\hline 48 & 84 & 69 & 90 & 87 & 81 & 42 \\
\hline 49 & 84 & 69 & 81 & 81 & 78 & 42 \\
\hline
\end{tabular}

Tab. 4. Total cost of multifunction execution units of the FFT algorithm

\begin{tabular}{|c|c|c|c|c|c|c|}
\hline$\propto$ & 文 & i⿱ & 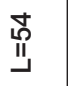 & 요 & 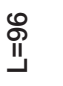 & $u^{\frac{c}{E}}$ \\
\hline 13 & 168 & 168 & 174 & 174 & 174 & 162 \\
\hline 14 & 180 & 180 & 174 & 171 & 177 & 150 \\
\hline 15 & 180 & 180 & 174 & 171 & 174 & 135 \\
\hline 16 & 186 & 186 & 186 & 186 & 171 & 123 \\
\hline 17 & 186 & 186 & 186 & 186 & 180 & 123 \\
\hline 18 & 186 & 186 & 186 & 183 & 180 & 108 \\
\hline 19 & 186 & 180 & 186 & 180 & 180 & 108 \\
\hline 20 & 183 & 183 & 174 & 180 & 180 & 108 \\
\hline 21 & 183 & 183 & 183 & 180 & 177 & 96 \\
\hline 22 & 180 & 174 & 180 & 180 & 177 & 96 \\
\hline 23 & 177 & 180 & 180 & 177 & 171 & 96 \\
\hline 24 & 180 & 177 & 174 & 177 & 171 & 81 \\
\hline 25 & 180 & 177 & 174 & 174 & 174 & 81 \\
\hline 26 & 180 & 177 & 171 & 162 & 147 & 81 \\
\hline 27 & 174 & 177 & 171 & 171 & 174 & 81 \\
\hline 28 & 165 & 165 & 150 & 156 & 138 & 81 \\
\hline 29 & 162 & 162 & 165 & 159 & 138 & 69 \\
\hline 30 & 138 & 138 & 138 & 105 & 102 & 69 \\
\hline 31 & 138 & 138 & 138 & 117 & 114 & 69 \\
\hline 32 & 129 & 129 & 129 & 108 & 105 & 69 \\
\hline 33 & 132 & 132 & 126 & 96 & 96 & 69 \\
\hline 34 & 120 & 120 & 111 & 108 & 105 & 69 \\
\hline 35 & 120 & 120 & 120 & 108 & 90 & 69 \\
\hline 36 & 120 & 120 & 120 & 108 & 102 & 54 \\
\hline 37 & 120 & 108 & 108 & 111 & 87 & 54 \\
\hline 38 & 120 & 120 & 120 & 108 & 93 & 54 \\
\hline 39 & 108 & 108 & 117 & 108 & 93 & 54 \\
\hline 40 & 120 & 108 & 108 & 108 & 102 & 54 \\
\hline 41 & 108 & 108 & 105 & 102 & 90 & 54 \\
\hline 42 & 108 & 117 & 105 & 102 & 87 & 54 \\
\hline 43 & 96 & 93 & 102 & 102 & 87 & 54 \\
\hline 44 & 96 & 93 & 102 & 105 & 84 & 54 \\
\hline 45 & 72 & 78 & 81 & 93 & 99 & 54 \\
\hline 46 & 72 & 69 & 78 & 81 & 90 & 54 \\
\hline 47 & 72 & 69 & 78 & 81 & 93 & 54 \\
\hline 48 & 72 & 81 & 78 & 66 & 81 & 42 \\
\hline 49 & 72 & 84 & 63 & 75 & 63 & 42 \\
\hline
\end{tabular}

